\title{
EDITORIAL
}

nature

cell biology

\section{All systems go!}

\section{Systems biology: a user's guide}

Systems biology is en vogue - the catch-phrase crops up in laboratories, grant applications and editorial offices in all manner of contexts. So what exactly is systems biology? Molecular cell biology encompasses a growing number of mechanistic but largely isolated insights and, increasingly, high-throughput 'omics' data sets; both are generally semi-quantitative and specific to a particular experimental system. The challenge is to integrate this complex and highly diverse information into a conceptual framework - one that is holistic, quantitative and predictive. One day, this might result in a 'virtual cell. Molecular cell biology seems to be on the verge of emancipating itself from a rather informal and purely reductionist hypothesis-driven approach by embracing high-throughput data acquisition, rigorous quantitation and mathematical modelling. In our opinion, the term systems biology describes this transition. In collaboration with Nature Reviews Molecular Cell Biology, we present a collection of articles to provide an introduction to and overview of systems approaches to molecular cell biology. The articles are light on jargon and technical detail, and they are infused with introductions to different methods, resources and glossary terms so as to make them accessible to the non-specialist reader.

Of course, informal static flowchart-type models have always formed the backbone of molecular biology; equally, theoretical biology is hardly new. However, systems biology describes the marriage of the two: it encompasses formal mathematical and computational modelling based on quantitative empirical data. It makes flow diagrams dynamic by adding quantitative information about changes to components in space and time. The outcome is not merely a more refined picture, but offers a new level of mechanistic understanding. Importantly, systems biology is not purely theoretical: it relies on experimental verification of its predictions. One key challenge is how to collect large volumes of complex data (see Albeck et al.) and organize them in a way that makes the data accessible to computational interrogation, as discussed by Kersey and Apweiler. Advanced modelling relies on highly structured systematic data sets, a topic discussed by Swedlow et al. using imaging data as an example. Large data sets can be analysed by data-driven modelling approaches (Yaffe and Janes), whereas 'physicochemical' and biophysical approaches are reviewed by Aldridge et al. and Karsenti et al. Modelling can also involve statistical approaches to identify meaningful data correlations, but, as discussed by Jaqaman and Danuser, linking models to data in a rigorous manner is not trivial.

Some argue that systems biology promises much, yet so far has delivered little; indeed, some bench scientists view the considerable hoopla around systems biology with some cynicism, probably secretly rubbing their hands gleefully when another modelling study concludes with rather mundane predictions not surpassing good intuition.
However, systems biology is a field in its infancy, constrained by limited data. It would be naive to dismiss too readily nascent modelling efforts that can end up with multiple variables and limited predictive power, as experimental and computational techniques are rapidly advancing. For example, dynamic imaging in live cells generates quantitative physiologically relevant data that takes the complexity of cellular structure into account, but that cannot be interpreted by simple inspection alone. In these cases, modelling and 'model friendly' experimentation work hand-in-hand. The aim of both is to improve the quantitative nature and reliability of data and their rigorous interpretation.

Systems biology is essential if we are ever to make sense of biological complexity, as intuitive 'conceptual' models quickly reach their limits beyond simple linear pathways. The time has come for molecular cell biologists, computer scientists and mathematicians to embrace each other's approaches, as is commonplace in the physical sciences. Collaborations are being successfully pursued, but cultural and philosophical barriers still need to be dismantled. More importantly, there is an urgent need to make the next generation of molecular cell biologists 'systems savvy'. The traditional segregation in higher education of biology from mathematics and physics presents a problem; Wingreen and Botstein discuss the educational challenges and propose an integration of these subjects for the biologists of the future.

Systems biology is making in-roads into every cell-biological discipline - far from being a passing fad, it marks the coming of age of molecular cell biology. Peter Sorger, a cell biologist who has made the transition to systems biology, sums it up: "Few fields of biology have escaped the influence of molecular approaches, and we anticipate that within a decade or two, this will also be true of systems approaches. Interest in systems biology reflects a genuine inability of informal conceptual modelling to tackle the sheer complexity of life."

We are pleased to acknowledge Pfizer, The MathWorks, Merrimack, Novartis and Entelos for their financial support. This Focus and accompanying reprint supplement were produced in association with the U.S. National Centers for Systems Biology as well as the journal Molecular Systems Biology. As always, Nature Publishing Group carries sole responsibility for all editorial content. The articles are freely available for one year. The articles are distributed between this issue and Nature Reviews Molecular Cell Biology and all articles can be accessed in a printed supplement or through a dedicated website (http://www.nature.com/focus/systemsbiologyuserguide), which also includes a library of recent systems biology papers across the Nature family of journals, lists of recommended text books and systems biology centres, and a podcast of discussion and interviews with several of the contributors to Systems biology: a user's guide. 